\title{
Comparative effectiveness of angiotensin-converting-enzyme inhibitors and angiotensin II receptor blockers in patients with type 2 diabetes and retinopathy
}

\author{
Chia-Jen Shih MD, Hung-Ta Chen MD, Shu-Chen Kuo MD, Szu-Yuan Li MD, Pi-Hsiang Lai BScN, \\ Shu-Chen Chen BScN, Shuo-Ming Ou MD, Yung-Tai Chen MD
}

Competing interests: None declared.

This article has been peer reviewed.

Accepted: Jan. 26, 2016

Online: Mar. 21, 2016

Correspondence to:

Yung-Tai Chen, ytchen0117@gmail.com; Shuo-Ming Ou, okokyytt@gmail.com

CMAJ 2016. DOI:10.1503/ cmaj.150771

\section{ABSTRACT}

Background: Angiotensin-converting-enzyme (ACE) inhibitors and angiotensin II receptor blockers (ARBs) are effective treatments for diabetic retinopathy, but randomized trials and meta-analyses comparing their effects on macrovascular complications have yielded conflicting results. We compared the effectiveness of these drugs in patients with pre-existing diabetic retinopathy in a large populationbased cohort.

Methods: We conducted a propensity scorematched cohort study using Taiwan's National Health Insurance Research Database. We included adult patients prescribed an ACE inhibitor or ARB within 90 days after diagnosis of diabetic retinopathy between 2000 and 2010 . Primary outcomes were all-cause death and major adverse cardiovascular events (myocardial infarction, ischemic stroke or cardiovascular death). Secondary outcomes were hospital admissions with acute kidney injury or hyperkalemia.
Results: We identified 11246 patients receiving ACE inhibitors and 15173 receiving ARBs, of whom 9769 patients in each group were matched successfully by propensity scores. In the intention-to-treat analyses, ARBs were similar to ACE inhibitors in risk of all-cause death (hazard ratio [HR] 0.94, 95\% confidence interval [Cl] 0.871.01) and major adverse cardiovascular events (HR 0.95, 95\% Cl 0.87-1.04), including myocardial infarction (HR 1.03, 95\% Cl 0.88-1.20), ischemic stroke (HR 0.94, 95\% Cl 0.85-1.04) and cardiovascular death (HR 1.01, 95\% Cl 0.88-1.16). They also did not differ from ACE inhibitors in risk of hospital admission with acute kidney injury (HR 1.01, 95\% Cl 0.91-1.13) and hospital admission with hyperkalemia (HR 1.01, 95\% Cl 0.86-1.18). Results were similar in as-treated analyses.

Interpretation: Our study showed that ACE inhibitors were similar to ARBs in risk of all-cause death, major adverse cardiovascular events and adverse effects among patients with preexisting diabetic retinopathy.
$\mathrm{D}$ iabetic retinopathy is among the most common microvascular complications in patients with type 2 diabetes and the leading cause of blindness in adults. The risk of incident macrovascular events is about 1.7- to 2.3-fold higher among patients with diabetic retinopathy than among those without it. ${ }^{1-3}$ Blockade of the renin-angiotensin-aldosterone system with angiotensin-converting-enzyme (ACE) inhibitors or angiotensin II receptor blockers (ARBs) is considered effective treatment for the prevention or regression of diabetic retinopathy, despite achieving only a modest decrease in blood pressure. ${ }^{4,5}$ In addition, given the microvascular and macrovascular benefits of these drugs, several relevant guidelines have recommended their use for first-line treatment of hypertension in patients with type 2 diabetes. ${ }^{6,7}$

The landmark Heart Outcomes Prevention Evaluation (HOPE) study ${ }^{8}$ found that use of
ACE inhibitors significantly reduced the risk of macrovascular events and composite microvascular events (progression of diabetic retinopathy requiring laser treatment, and overt nephropathy) among patients with type 2 diabetes and vascular disease, compared with placebo. Angiotensinreceptor blockers that selectively inhibit angiotensin II type 1 receptors theoretically offer more specific inhibition of the renin-angiotensinaldosterone system and have fewer adverse systemic effects than ACE inhibitors. In a post-hoc analysis conducted as part of the Diabetic Retinopathy Candesartan Trials of the effect of candesartan on progression and regression of retinopathy in type 2 diabetes (DIRECT-Protect 2 study), ${ }^{9}$ ARBs appeared to reduce the risk of macrovascular complications in patients with diabetic retinopathy compared with placebo, although the results were not statistically significant. Other studies have documented the reno- 
protective benefits of ARBs in patients with type 2 diabetes and nephropathy, ${ }^{10,11}$ but whether these drugs have cardioprotective effects similar to those of ACE inhibitors remains unclear. ${ }^{12,13}$

Several meta-analyses have compared the effectiveness of ACE inhibitors and ARBs in diabetic populations, ${ }^{14,15}$ but they have produced conflicting results, probably owing to heterogeneity among trials, differences in enrolment criteria used in clinical trials and differences in the baseline burden of diabetes between the ACE inhibitor and ARB groups. In the Ongoing Telmisartan Alone and in Combination with Ramipril Global End point (ONTARGET) trial, ${ }^{16}$ evidence from the diabetes subgroup (38\% of the study cohort, with evidence of end-organ damage) showed that ARBs were not inferior to ACE inhibitors in terms of major adverse cardiac events. However, previous studies involved diabetic patients with different disease processes, and thus the available evidence is not sufficient to determine the relative appropriateness of ACE inhibitors and ARBs for the prevention of macrovascular disease in patients with pre-existing diabetic retinopathy, who represent a more homogeneous population at high cardiovascular risk.

Given the paucity of head-to-head trials to bridge this evidence gap, we compared the effectiveness of ACE inhibitors and ARBs on major adverse cardiac events in a nationwide, propensity score-matched, population-based cohort of patients with diabetic retinopathy.

\section{Methods}

\section{Study population and design}

We used the Longitudinal Cohort of Diabetes Patients dataset, extracted from Taiwan's National Health Insurance Research Database (NHIRD). This database contains detailed medical claims data from almost all of Taiwan's inhabitants (average 23 million) since 1995 and has been described in detail previously. ${ }^{17,18} \mathrm{We}$ used International Classification of Diseases, Ninth Revision, Clinical Modification (ICD9-CM) codes to identify important comorbid conditions. We first selected patients with 1 primary discharge diagnosis or 2 outpatient diagnoses of diabetes (ICD-9-CM code 250.x). The accuracy of diagnostic coding of diabetes in the NHIRD database has been validated previously. ${ }^{19}$ From this sample, we then selected all adults (age $\geq 20 \mathrm{yr}$ ) with diabetic retinopathy (ICD-9-CM code 362.0) diagnosed between January 2000 and December 2010, confirmed by ophthalmologists via funduscopic examination, according to the guidelines of the Early Treatment Diabetic Retinopathy Study. ${ }^{20,21}$
The Institutional Review Board of Taipei City Hospital exempted this study from full review because the NHIRD database contains encrypted and de-identified claims data released exclusively for research purposes.

\section{Study cohorts}

Eligible patients who received prescriptions for an ACE inhibitor or ARB within 90 days after diagnosis of their diabetic retinopathy were allocated to the ACE inhibitor and ARB cohorts. To avoid immortal time bias, we designated the index date as 91 days after diagnosis. We excluded patients who were switched to the other study drug or were prescribed a combination of ACE inhibitor and ARB within 90 days after diagnosis. To identify incident events of interest, we excluded patients who had myocardial infarction or cerebrovascular disease before the index date.

Because treatment with ACE inhibitors or ARBs may introduce confounding by indication, we used a propensity score analysis to control for any baseline imbalance between cohorts, including diseases and concomitant medications that may have biased the association between treatment and outcomes of interest. We matched each patient in the ACE inhibitor cohort to a patient in the ARB cohort with the closest propensity score using nearest-neighbour matching without replacement, and a caliper width of 0.1 of the standard deviation of the logit of the propensity score.

\section{Potential confounders}

For each patient, we extracted all medical data for the 5-year period before the index date from the NHIRD database to obtain information on medical history and medication use. Baseline demographic covariates included age, sex, year of index date, month of index date, monthly income, urbanization level, Charlson Comorbidity Index score and adapted Diabetes Complications Severity Index score. ${ }^{22}$ Information on concomitant use of other medications, including antidiabetic drugs, antihypertensive agents, antiplatelet agents, anticoagulants, nitrates, statins, proton-pump inhibitors and nonsteroidal anti-inflammatory drugs, was extracted. In addition, we identified major baseline comorbidities that were potentially associated with the outcomes of interest but were not included in the Charlson Comorbidity Index.

\section{Exposure to study drugs}

From the Longitudinal Cohort of Diabetes Patients dataset, we extracted information on all $\mathrm{ACE}$ inhibitor and $\mathrm{ARB}$ prescriptions during the study period, including drug name, quantity, dose, and start and stop dates. Drug persistence was defined as the duration of drug use, from ini- 
tiation to discontinuation. We allowed a grace period of 90 days between prescriptions when calculating individuals' drug persistence. We considered the first occurrence of a gap of more than 90 days after a previous prescription to represent the discontinuation of the medication.

\section{Outcomes}

The primary outcomes were all-cause death and major adverse cardiac events, including hospital admission with the principal diagnosis of myocardial infarction (ICD-9-CM code 410.x) or ischemic stroke (ICD-9-CM code 433.x, 434.x, or 436) and in-hospital cardiovascular death. The accuracy of diagnostic coding of myocardial infarction and ischemic stroke in the NHIRD database has been reported to be as high as $93 \%$ and $94 \%$, respectively. ${ }^{23,24}$ The secondary outcomes were hospital admission with the principal diagnosis of acute kidney injury (ICD-9-CM code 584.x) or hyperkalemia (ICD-9-CM code 276.7) during follow-up. All patients were followed until death or Dec. 31, 2012.

\section{Statistical analysis}

We calculated propensity scores for the likelihood of ARB use (with ACE inhibitor use as the

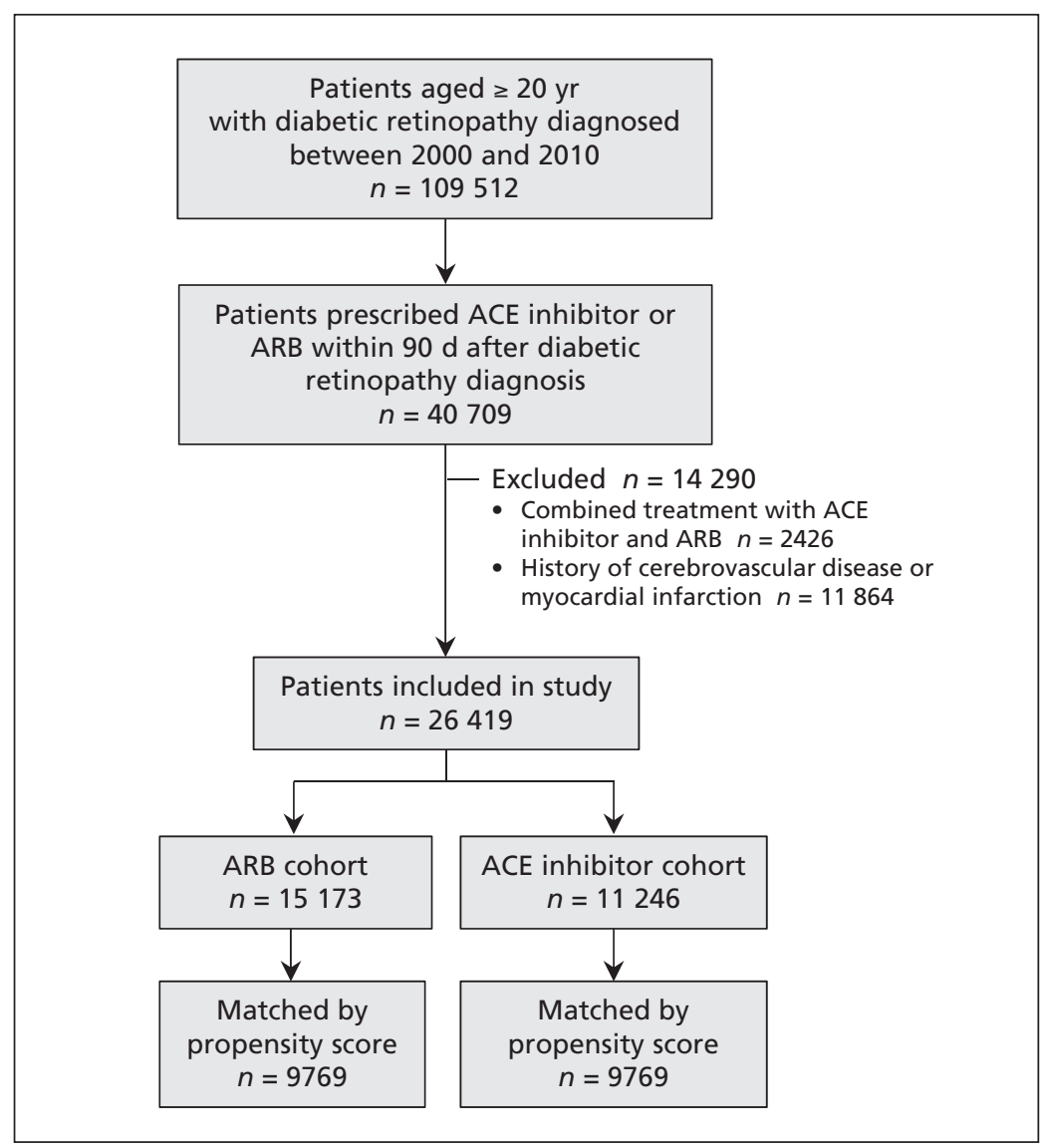

Figure 1: Selection of study cohorts. $A C E$ = angiotensin-converting enzyme, ARB = angiotensin II receptor blocker. reference) using logistic regression, conditional on predefined covariates (Appendix 1, available at www.cmaj.ca/lookup/suppl/doi:10.1503/ cmaj.150771/-/DC1). The standardized mean difference was used to compare groups before and after propensity score matching. Intentionto-treat analyses were used as the primary analyses for this study because of more reliable estimates of comparative treatment effectiveness in real-world applications. Both cohorts were followed until the study outcome according to original treatment allocation, regardless of adherence to or subsequent withdrawal or deviation from the inclusion criteria.

We determined the cumulative incidence of events of interest using the Kaplan-Meier method and compared between-group rates using the log-rank test. We used a Cox regression model to calculate hazard ratios (HRs) with $95 \%$ confidence intervals (CIs) for the risks of events of interest. The likelihood ratio test was used to test for interaction with select covariates (age, sex, Charlson Comorbidity Index score, hypertension, chronic kidney disease, heart failure and coronary artery disease), and subgroup analyses were performed accordingly. We considered an interaction term to be significant if Bonferronicorrected alpha levels were $0.007(0.05 / 7)$.

Finally, we performed several sensitivity analyses, which included as-treated analyses, Cox analyses with death serving as a competing risk, analyses before propensity score matching, and analyses excluding ACE inhibitor or ARB use before the diagnosis of diabetic retinopathy. In the as-treated analyses, the person-time in the as-treated population, a subset of all person-time in the intention-totreat analyses, was censored on the day of medication add-on, switching or discontinuation. For the Cox model with death serving as a competing risk, we used the Fine and Gray method. ${ }^{25}$

We used the Microsoft SQL Server 2012 (Microsoft Corporation) for data linkage, processing and sampling. We calculated propensity scores using SAS version 9.3 (SAS Institute Inc.). All other statistical analyses were conducted using STATA statistical software version 13.0 (StataCorp). Statistical significance was defined as $p<0.05$.

\section{Results}

\section{Patient characteristics}

We identified 26419 patients with diabetic retinopathy who were prescribed an ARB $(n=$ $15173)$ or an ACE inhibitor ( $n=11246)$ within 90 days after their diabetic retinopathy was diagnosed (Figure 1). Table 1 shows the demographic and clinical characteristics of the 
Table 1 (part 1 of 2): Baseline characteristics of patients with diabetic retinopathy prescribed ARBs and ACE inhibitors, before and after matching by propensity scores

\begin{tabular}{|c|c|c|c|c|c|c|}
\hline \multirow[b]{2}{*}{ Characteristic } & \multicolumn{3}{|c|}{ Before matching; no. (\%) of patients* } & \multicolumn{3}{|c|}{ After matching; no. (\%) of patients* } \\
\hline & $\begin{array}{l}\text { ARB cohort } \\
n=15173\end{array}$ & $\begin{array}{c}\text { ACE inhibitor } \\
\text { cohort } \\
n=11246\end{array}$ & $\begin{array}{l}\text { Standardized } \\
\text { difference, } † \%\end{array}$ & $\begin{array}{l}\text { ARB cohort } \\
n=9769\end{array}$ & $\begin{array}{c}\text { ACE inhibitor } \\
\text { cohort } \\
n=9769\end{array}$ & $\begin{array}{l}\text { Standardized } \\
\text { difference, } \dagger \%\end{array}$ \\
\hline Age, yr, mean \pm SD & $59.7 \pm 11.1$ & $58.6 \pm 11.2$ & 0.091 & $58.9 \pm 11.1$ & $59.0 \pm 11.2$ & -0.005 \\
\hline Sex, male & $7276(48.0)$ & $6044(53.7)$ & -0.116 & $5112(52.3)$ & $5075(52.0)$ & 0.008 \\
\hline \multicolumn{7}{|l|}{ Year of index date } \\
\hline 2000 & $117 \quad(0.8)$ & $288 \quad(2.6)$ & -0.140 & $117 \quad(1.2)$ & $111 \quad(1.1)$ & 0.006 \\
\hline 2001 & $210 \quad(1.4)$ & $524 \quad(4.7)$ & -0.192 & $210 \quad(2.1)$ & $197 \quad(2.0)$ & 0.009 \\
\hline 2002 & $500 \quad(3.3)$ & $803 \quad(7.1)$ & -0.174 & $488 \quad(5.0)$ & $490 \quad(5.0)$ & -0.001 \\
\hline 2003 & $779 \quad(5.1)$ & $955 \quad(8.5)$ & -0.134 & $731 \quad(7.5)$ & $735 \quad(7.5)$ & -0.002 \\
\hline 2004 & 1160 & $1159(10.3)$ & -0.093 & $996(10.2)$ & $973(10.0)$ & 0.008 \\
\hline 2005 & $1365 \quad(9.0)$ & $1258(11.2)$ & -0.073 & $1122(11.5)$ & 1132 (11.6) & -0.003 \\
\hline 2006 & $1478 \quad(9.7)$ & $1235(11.0)$ & -0.041 & 1158 (11.9) & 1156 (11.8) & 0.001 \\
\hline 2007 & $1704(11.2)$ & $1158(10.3)$ & 0.030 & $1131(11.6)$ & $1126(11.5)$ & 0.002 \\
\hline 2008 & $2074(13.7)$ & $1260(11.2)$ & 0.075 & $1272(13.0)$ & $1245(12.7)$ & 0.008 \\
\hline 2009 & $2474(16.3)$ & $1264(11.2)$ & 0.147 & $1238(12.7)$ & $1262(12.9)$ & -0.007 \\
\hline 2010 & $3312(21.8)$ & 1342 (11.9) & 0.266 & 1306 (13.4) & $1342(13.7)$ & -0.011 \\
\hline \multicolumn{7}{|l|}{ Monthly income, NT\$ } \\
\hline Dependent & 4785 (31.5) & $3386(30.1)$ & 0.031 & $2965(30.4)$ & $2980(30.5)$ & -0.003 \\
\hline$<19100$ & $2897(19.1)$ & $2255(20.1)$ & -0.024 & $1946(19.9)$ & $1916(19.6)$ & 0.008 \\
\hline 19 100-41999 & 6360 (41.9) & $4855(43.2)$ & -0.025 & $4173(42.7)$ & $4201(43.0)$ & -0.006 \\
\hline$\geq 42000$ & 1131 & $750 \quad(6.7)$ & 0.031 & $685 \quad(7.0)$ & $672 \quad(6.9)$ & 0.005 \\
\hline \multicolumn{7}{|l|}{ Urbanization‡ } \\
\hline Level 1 (most urban) & $5833(38.4)$ & $4547(40.4)$ & -0.041 & $3909(40.0)$ & $3906(40.0)$ & 0.001 \\
\hline Level 2 & $8530(56.2)$ & 6156 (54.7) & 0.030 & $5367(54.9)$ & $5379(55.1)$ & -0.002 \\
\hline Level 3 & $699 \quad(4.6)$ & $458 \quad(4.1)$ & 0.026 & $419 \quad(4.3)$ & $408 \quad(4.2)$ & 0.006 \\
\hline Level 4 (least urban) & $111 \quad(0.7)$ & $85 \quad(0.8)$ & -0.003 & $74 \quad(0.8)$ & $76 \quad(0.8)$ & -0.002 \\
\hline \multicolumn{7}{|c|}{ Outpatient visits in the past year } \\
\hline$<6$ & $82 \quad(0.5)$ & $63 \quad(0.6)$ & -0.003 & $53 \quad(0.5)$ & $52 \quad(0.5)$ & 0.001 \\
\hline $6-10$ & $819 \quad(5.4)$ & $642 \quad(5.7)$ & -0.014 & $544 \quad(5.6)$ & $569 \quad(5.8)$ & -0.011 \\
\hline $11-15$ & $1944(12.8)$ & $1404(12.5)$ & 0.010 & $1262(12.9)$ & $1259(12.9)$ & 0.001 \\
\hline$>15$ & $12328(81.2)$ & $9137(81.2)$ & 0.000 & $7910(81.0)$ & $7889(80.8)$ & 0.005 \\
\hline \multicolumn{7}{|c|}{ Charlson Comorbidity Index score§ } \\
\hline 2 & $4105(27.1)$ & $3666(32.6)$ & -0.121 & $2999(30.7)$ & $3030(31.0)$ & -0.007 \\
\hline 3 & $2977(19.6)$ & $2445(21.7)$ & -0.052 & $2066(21.1)$ & $2049(21.0)$ & 0.004 \\
\hline 4 & $2663(17.6)$ & $1920(17.1)$ & 0.013 & $1711(17.5)$ & $1714(17.5)$ & -0.001 \\
\hline$\geq 5$ & $5428(35.8)$ & $3215(28.6)$ & 0.154 & $2993(30.6)$ & $2976(30.5)$ & 0.004 \\
\hline $\begin{array}{l}\text { Adapted DCSI score, } \emptyset \\
\text { mean } \pm \text { SD }\end{array}$ & $3.0 \pm 1.9$ & $2.8 \pm 1.8$ & 0.135 & $2.8 \pm 1.8$ & $2.8 \pm 1.8$ & 0.001 \\
\hline \multicolumn{7}{|l|}{ Antidiabetic drug use } \\
\hline Acarbose & $1576(10.4)$ & $855 \quad(7.6)$ & 0.097 & $855 \quad(8.8)$ & $821 \quad(8.4)$ & 0.012 \\
\hline Sulfonylurea & $7736(51.0)$ & 5819 (51.7) & -0.015 & $4993(51.1)$ & $5058(51.8)$ & -0.013 \\
\hline Insulin & $907 \quad(6.0)$ & $595 \quad(5.3)$ & 0.030 & $528 \quad(5.4)$ & $527 \quad(5.4)$ & 0.000 \\
\hline Metformin & 9395 (61.9) & $6958(61.9)$ & 0.001 & $6072(62.2)$ & $6071(62.1)$ & 0.000 \\
\hline Thiazolidinedione & $2304(15.2)$ & 1038 & 0.183 & $999(10.2)$ & $1022(10.5)$ & -0.008 \\
\hline Glinide & $1092 \quad(7.2)$ & $(5.0)$ & 0.090 & $575 \quad(5.9)$ & $547 \quad(5.6)$ & 0.012 \\
\hline DPP-4 inhibitor & $529 \quad(3.5)$ & $103 \quad(0.9)$ & 0.176 & $127 \quad(1.3)$ & $103 \quad(1.1)$ & 0.023 \\
\hline
\end{tabular}


Table 1 (part 2 of 2): Baseline characteristics of patients with diabetic retinopathy prescribed ARBs and ACE inhibitors, before and after matching by propensity scores

\begin{tabular}{|c|c|c|c|c|c|c|}
\hline \multirow[b]{2}{*}{ Characteristic } & \multicolumn{3}{|c|}{ Before matching; no. (\%) of patients* } & \multicolumn{3}{|c|}{ After matching; no. (\%) of patients* } \\
\hline & $\begin{array}{l}\text { ARB cohort } \\
n=15173\end{array}$ & $\begin{array}{c}\text { ACE inhibitor } \\
\text { cohort } \\
n=11246\end{array}$ & $\begin{array}{l}\text { Standardized } \\
\text { difference, } † \%\end{array}$ & $\begin{array}{l}\text { ARB cohort } \\
n=9769\end{array}$ & $\begin{array}{c}\text { ACE inhibitor } \\
\text { cohort } \\
n=9769\end{array}$ & $\begin{array}{l}\text { Standardized } \\
\text { difference, } \uparrow \%\end{array}$ \\
\hline \multicolumn{7}{|l|}{ Antihypertensive drug use } \\
\hline$\alpha$-Blocker & $565 \quad(3.7)$ & $372 \quad(3.3)$ & 0.023 & $335 \quad(3.4)$ & $326 \quad(3.3)$ & 0.005 \\
\hline$\beta$-Blocker & $2693(17.7)$ & $1874(16.7)$ & 0.029 & $1683(17.2)$ & $1625(16.6)$ & 0.016 \\
\hline Calcium-channel blocker & $6321(41.7)$ & $3833(34.1)$ & 0.157 & $3576(36.6)$ & 3488 (35.7) & 0.019 \\
\hline Diuretic & $2715(17.9)$ & $2032(18.1)$ & -0.005 & $1762(18.0)$ & $1761(18.0)$ & 0.000 \\
\hline Other & $178 \quad(1.2)$ & $213 \quad(1.9)$ & -0.059 & $140 \quad(1.4)$ & $150 \quad(1.5)$ & -0.008 \\
\hline \multicolumn{7}{|l|}{ Other medication use } \\
\hline ASA & 3101 (20.4) & $2031(18.1)$ & 0.060 & $1850(18.9)$ & $1814(18.6)$ & 0.009 \\
\hline Clopidogrel & $164(1.1)$ & $52 \quad(0.5)$ & 0.071 & $51 \quad(0.5)$ & $52 \quad(0.5)$ & -0.001 \\
\hline Ticlopidine & $71 \quad(0.5)$ & $30 \quad(0.3)$ & 0.033 & $30 \quad(0.3)$ & $29 \quad(0.3)$ & 0.002 \\
\hline Warfarin & $78 \quad(0.5)$ & $40 \quad(0.4)$ & 0.024 & $38 \quad(0.4)$ & $37 \quad(0.4)$ & 0.002 \\
\hline Dipyridamole & $760 \quad(5.0)$ & $(5.3)$ & -0.014 & $(4.9)$ & $488 \quad(5.0)$ & -0.005 \\
\hline Nitrate & $739 \quad(4.9)$ & $479 \quad(4.3)$ & 0.029 & $423 \quad(4.3)$ & $408 \quad(4.2)$ & 0.008 \\
\hline Statin & $4161(27.4)$ & $2349(20.9)$ & 0.153 & $2226(22.8)$ & $2217(22.7)$ & 0.002 \\
\hline Proton pump inhibitor & $315 \quad(2.1)$ & $173 \quad(1.5)$ & 0.040 & $158 \quad(1.6)$ & 159 (1.6) & -0.001 \\
\hline NSAID & $2251(14.8)$ & $1928(17.1)$ & -0.063 & $1580(16.2)$ & $1581(16.2)$ & -0.000 \\
\hline \multicolumn{7}{|l|}{ Comorbidities } \\
\hline Hypertension & $14299(94.2)$ & $10271(91.3)$ & 0.113 & $9037(92.5)$ & $9030(92.4)$ & 0.003 \\
\hline Coronary artery disease & 5559 (36.6) & 3532 (31.4) & 0.111 & $3223(33.0)$ & 3204 (32.8) & 0.004 \\
\hline Heart failure & $1726(11.4)$ & $991 \quad(8.8)$ & 0.085 & $904 \quad(9.3)$ & $903 \quad(9.2)$ & 0.000 \\
\hline Peripheral vascular disease & $809 \quad(5.3)$ & $587 \quad(5.2)$ & 0.005 & $520 \quad(5.3)$ & $512 \quad(5.2)$ & 0.004 \\
\hline Peptic ulcer disease & 6211 (40.9) & $4071(36.2)$ & 0.097 & 3709 (38.0) & $3717(38.0)$ & -0.002 \\
\hline Liver disease & 5266 (34.7) & $3625(32.2)$ & 0.052 & 3275 (33.5) & 3262 (33.4) & 0.003 \\
\hline Chronic kidney disease & 2957 (19.5) & 1756 (15.6) & 0.102 & 1589 (16.3) & $1600(16.4)$ & -0.003 \\
\hline Atrial fibrillation & $354 \quad(2.3)$ & $176 \quad(1.6)$ & 0.056 & $176 \quad(1.8)$ & $170 \quad(1.7)$ & 0.005 \\
\hline Dyslipidemia & $10432(68.8)$ & $7073(62.9)$ & 0.124 & $6343(64.9)$ & $6388(65.4)$ & -0.010 \\
\hline Valvular heart disease & $1155 \quad(7.6)$ & $635 \quad(5.6)$ & 0.079 & $601 \quad(6.2)$ & $598 \quad(6.1)$ & 0.001 \\
\hline Cancer & $1400 \quad(9.2)$ & $832 \quad(7.4)$ & 0.066 & $788 \quad(8.1)$ & $773 \quad(7.9)$ & 0.006 \\
\hline Autoimmune disease & $593 \quad(3.9)$ & $346 \quad(3.1)$ & 0.045 & $306 \quad(3.1)$ & $328 \quad(3.4)$ & -0.013 \\
\hline Physical limitation & $727 \quad(4.8)$ & $496 \quad(4.4)$ & 0.018 & $443 \quad(4.5)$ & $451 \quad(4.6)$ & -0.004 \\
\hline Propensity score, mean \pm SD & $0.61 \pm 0.13$ & $0.53 \pm 0.14$ & 0.590 & $0.56 \pm 0.12$ & $0.56 \pm 0.12$ & 0.002 \\
\hline \multicolumn{7}{|c|}{$\begin{array}{l}\text { ACE inhibitor or ARB use before } \\
\text { diabetic retinopathy diagnosis }\end{array}$} \\
\hline ACE inhibitor use & $549 \quad(3.6)$ & $6168(54.8)$ & & $319 \quad(3.3)$ & $4781(48.9)$ & \\
\hline ARB use & $9927(65.4)$ & $200 \quad(1.8)$ & & $5023(51.4)$ & $163(1.7)$ & \\
\hline \multicolumn{7}{|c|}{$\begin{array}{l}\text { Note: ACE = angiotensin-converting enzyme, ARB = angiotensin II receptor blocker, ASA = acetylsalicylic acid, DCSI = Diabetes Complications Severity Index, } \\
\text { DPP = dipeptidyl peptidase, NSAID = nonsteroidal anti-inflammatory drug, NT\$ = new Taiwan dollars. } \\
\text { *Unless stated otherwise. } \\
\text { tImbalance defined as absolute value greater than } 0.024 \text { for unmatched cohorts and greater than } 0.028 \text { for matched cohorts. } \\
\text { fUrbanization levels in Taiwan are divided into } 4 \text { strata according to Taiwan National Health Research Institute publications. Level } 1 \text { designates the most } \\
\text { urbanized areas, and level } 4 \text { designates the least urbanized areas. } \\
\text { \$Charlson Comorbidity Index score is used to determine overall systemic health. With each increased level of the score, there are stepwise increases in the } \\
\text { cumulative mortality. } \\
\text { IAdapted Diabetes Complications Severity Index is a 13-point scale derived from } 7 \text { complication categories (retinopathy, nephropathy, neuropathy, } \\
\text { cerebrovascular, cardiovascular, peripheral vascular disease and metabolic). Each complication produces a numeric score ranging from } 0 \text { to } 2(0=\text { no abnormality, } 1 \\
=\text { some abnormality, } 2=\text { severe abnormality). }\end{array}$} \\
\hline
\end{tabular}


patients before and after propensity score matching. In the full cohort, compared with patients taking ACE inhibitors, those taking ARBs were older and predominantly female and were more likely to have higher Charlson Comorbidity Index and adapted Diabetes Complications Severity Index scores and to have more comorbidities (e.g., hypertension, coronary artery disease, heart failure, chronic kidney disease, atrial fibrillation, dyslipidemia, valvular heart disease and cancer). Using propensity scores, we were able to match 9769 patients in the ACE inhibitor cohort with 9769 in the ARB cohort. There was no meaningful difference in baseline characteristics between the matched groups (Table 1; Appendix 2, available at www.cmaj.ca/lookup/ suppl/doi:10.1503/cmaj.150771///DC1). The differential characteristics between the matched and unmatched patients in both groups are also illustrated in Appendix 3 (available at www.cmaj.ca/ lookup/suppl/doi:10.1503/cmaj.150771/-/DC1).

\section{Effect on outcomes}

The mean follow-up period was 72.2 months in the ACE inhibitor group and 60.2 months in the ARB group. Continuous use of ACE inhibitors at 1,3 and 5 years was recorded for $64.6 \%$, $29.9 \%$ and $20.7 \%$, respectively, of patients in the ACE inhibitor group; corresponding values for continuous use of ARBs were $82.6 \%, 44.7 \%$ and $43.8 \%$ of patients in the ARB group. During the follow-up period, the proportion of patients who were switched to the other study drug or were given both drugs was lower in the ARB group (9.2\% switched and $18.5 \%$ added an ACE inhibitor) than in the ACE inhibitor group (40.1\% switched and $23.5 \%$ added an ARB).

In the full cohort, the crude event rates per
1000 person-years (ARBs v. ACE inhibitors) were 25.6 v. 28.7 for all-cause death, 17.3 v. 19.1 for major adverse cardiac events (including 5.6 v. 5.7 for myocardial infarction, 12.4 v. 13.8 for ischemic stroke and $8.0 \mathrm{v}$. 7.7 for cardiovascular death), 13.1 v. 12.2 for hospital admission with acute kidney injury and 6.1 v. 5.9 for hospital admission with hyperkalemia. In the intention-to-treat analyses conducted after propensity score matching, the event rates per 1000 personyears in the ARB cohort were similar to those in the ACE inhibitor cohort: 26.0 v. 27.7 for allcause death (HR 0.94, 95\% CI 0.87-1.01), 18.2 v. 19.0 for major adverse cardiac events (HR $0.95,95 \%$ CI $0.87-1.04$ ), including 5.9 v. 5.7 for myocardial infarction (HR 1.03, 95\% CI 0.88 1.20), 13.0 v. 13.9 for ischemic stroke (HR 0.94, 95\% CI $0.85-1.04)$ and 7.6 v. 7.5 for cardiovascular death (HR 1.01, 95\% CI 0.88-1.16) (Table 2). The rates per 1000 person-years of the secondary outcomes were also similar between the ARB and ACE inhibitor cohorts (Table 2).

We found no difference in the cumulative incidence rates of all-cause death and major adverse cardiac events (Figure 2) or in adverse effects (Appendix 4, available at www.cmaj.ca/lookup/ suppl/doi:10.1503/cmaj.150771/-/DC1). The outcomes were also similar between cohorts in the subgroup analyses defined according to age, sex, comorbidities and baseline cardiovascular risk burden (Appendices 5-8, available at www.cmaj.ca/ lookup/suppl/doi:10.1503/cmaj.150771/-/DC1).

We obtained similar results in the sensitivity analyses (Figure 3, and Appendices 9 and 10, available at www.cmaj.ca/lookup/suppl/ doi:10.1503/cmaj.150771/-/DC1). In the astreated analyses, the 2 patient groups had similar risks of major adverse cardiac events and adverse

Table 2: Event rates of primary and secondary outcomes in a matched cohort of patients with diabetic retinopathy prescribed ARBs and ACE inhibitors

\begin{tabular}{|c|c|c|c|c|c|c|c|}
\hline \multirow[b]{2}{*}{ Outcome } & \multicolumn{3}{|c|}{ ARB cohort } & \multicolumn{3}{|c|}{ ACE inhibitor cohort (reference) } & \multirow[b]{2}{*}{$\begin{array}{l}\text { Hazard ratio } \\
(95 \% \mathrm{Cl})\end{array}$} \\
\hline & $\begin{array}{l}\text { No. of } \\
\text { events }\end{array}$ & $\begin{array}{c}\text { No. of } \\
\text { person-years }\end{array}$ & $\begin{array}{l}\text { Incidence } \\
\text { rate* }\end{array}$ & $\begin{array}{l}\text { No. of } \\
\text { events }\end{array}$ & $\begin{array}{c}\text { No. of } \\
\text { person-years }\end{array}$ & $\begin{array}{l}\text { Incidence } \\
\text { rate* }\end{array}$ & \\
\hline All-cause death & 1421 & 54620 & 26.0 & 1500 & 54129 & 27.7 & $0.94(0.87-1.01)$ \\
\hline Major adverse cardiac events & 944 & 52004 & 18.2 & 978 & 51409 & 19.0 & $0.95(0.87-1.04)$ \\
\hline Cardiovascular death & 417 & 54581 & 7.6 & 408 & 54103 & 7.5 & $1.01(0.88-1.16)$ \\
\hline $\begin{array}{l}\text { Hospital admission with } \\
\text { acute kidney injury }\end{array}$ & 671 & 53311 & 12.6 & 656 & 52829 & 12.4 & $1.01(0.91-1.13)$ \\
\hline $\begin{array}{l}\text { Hospital admission with } \\
\text { hyperkalemia }\end{array}$ & 320 & 53892 & 5.9 & 314 & 53325 & 5.9 & $1.01(0.86-1.18)$ \\
\hline
\end{tabular}



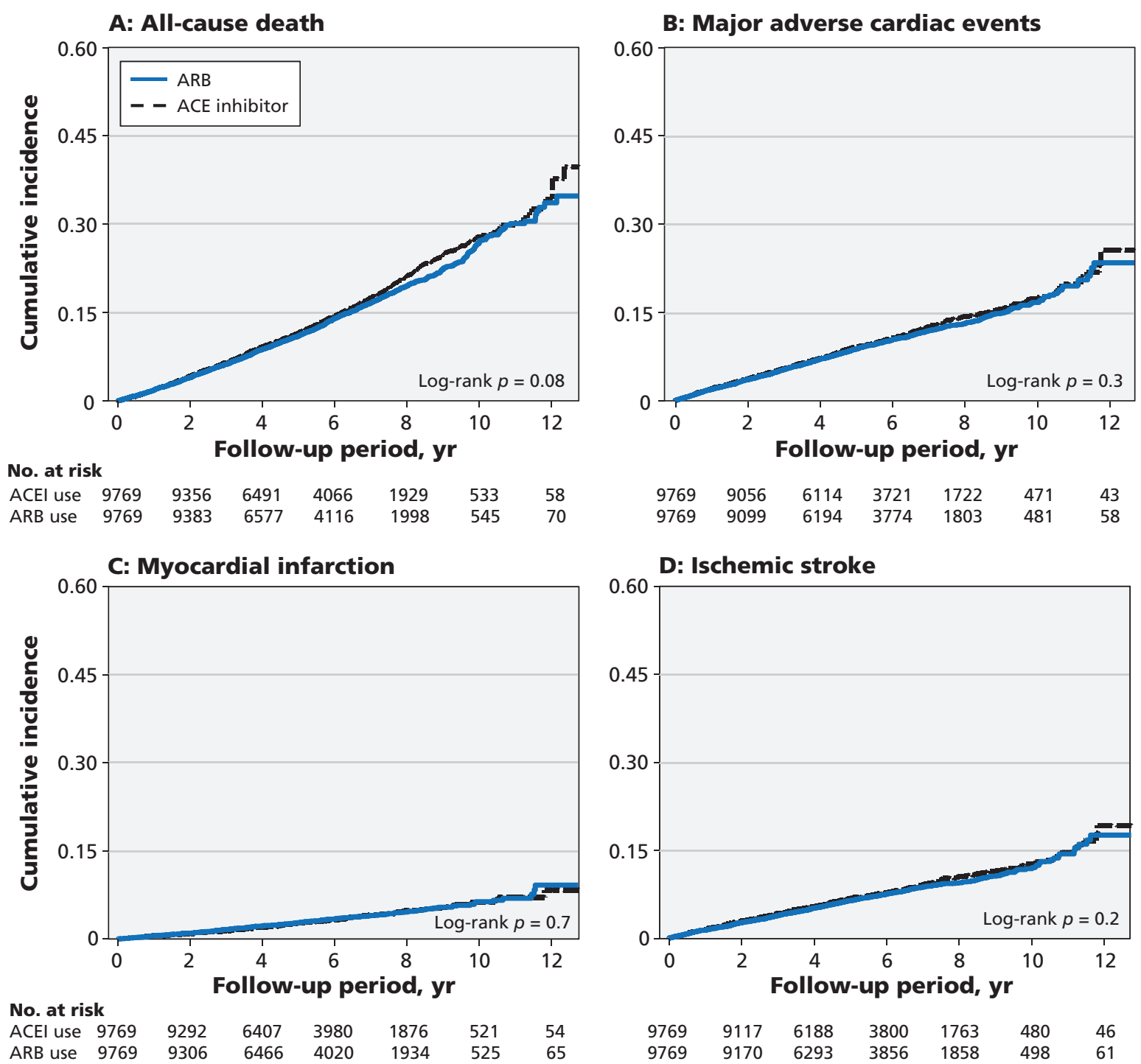

No. at risk

\begin{tabular}{|c|c|c|c|c|c|c|}
\hline & 9769 & 9292 & 6407 & 3980 & 1876 & 521 \\
\hline ARB us & 9769 & 9306 & 646 & 4020 & 1934 & 525 \\
\hline
\end{tabular}

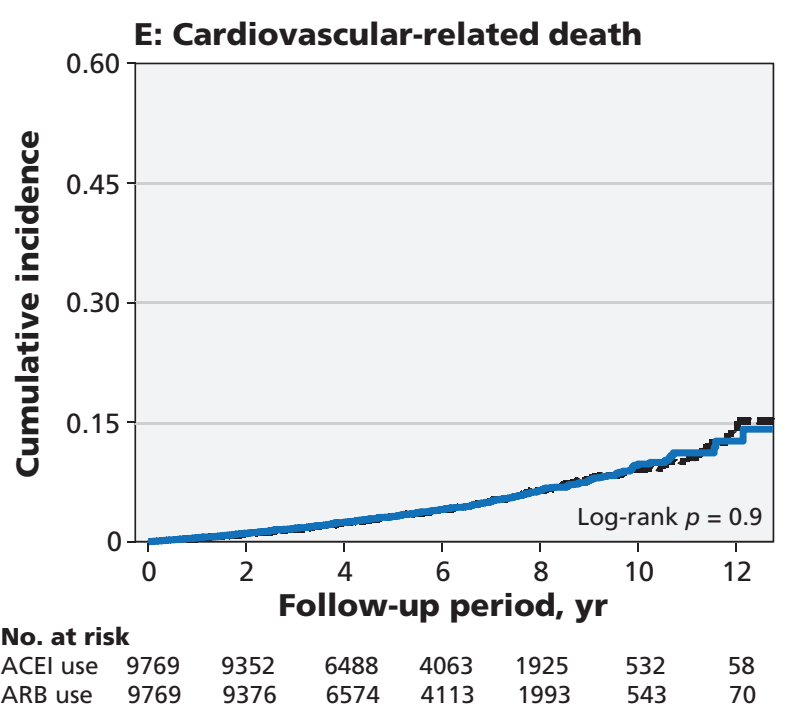

Figure 2: Cumulative incidence of (A) all-cause death, (B) major adverse cardiac events, (C) myocardial infarction, (D) ischemic stroke and (E) cardiovascular death among patients with diabetic retinopathy prescribed angiotensin-converting-enzyme inhibitor (ACEI) or angiotensin II receptor blocker (ARB). 
effects. When death was treated as a competing risk, the risks of major adverse cardiac events and hospital admission with hyperkalemia were similar between patient groups, although the ARB cohort had a higher risk of hospital admission with acute kidney injury (HR 1.27, 95\% CI 1.051.55). Analyses conducted before propensity score matching produced consistent results with those after adjustment for propensity scores. After we excluded patients with prevalent ACE inhibitor or ARB exposure, the results remained unchanged after adjustment for propensity score (Appendix 11, available at www.cmaj.ca/lookup/ suppl/doi:10.1503/cmaj.150771/-/DC1).

\section{Interpretation}

In this study, we found no difference in the risk of all-cause death, major adverse cardiac events and adverse effects (hospital admission with acute kidney injury or hyperkalemia) between patients with diabetic retinopathy prescribed ACE inhibitors and those prescribed ARBs. Similar findings were obtained in subgroup analyses by age, sex and baseline cardiovascular risk burden.

To our knowledge, no head-to-head randomized controlled trial (RCT) has examined the effects of ACE inhibitors and ARBs in patients with pre-existing diabetic retinopathy. In a recent meta-analysis that indirectly compared reninangiotensin-aldosterone system blockers, ${ }^{26} \mathrm{ACE}$ inhibitors appeared to be superior to ARBs for the treatment of diabetic retinopathy, but analyses were not sufficiently powered to detect a significant difference in incident macrovascular events in diabetic retinopathy trials. Previous small RCTs ${ }^{27-31}$ directly comparing the effectiveness of ACE inhibitors and ARBs focused mainly on renal outcomes in patients with type 2 diabetes and albuminuria; they revealed no difference during short follow-up periods. Similar results for major adverse cardiac events were observed in the diabetes subgroup of a large ONTARGET trial, ${ }^{16}$ but randomization was not based on the presence and severity of diabetes, and baseline diabetic retinopathy status was not

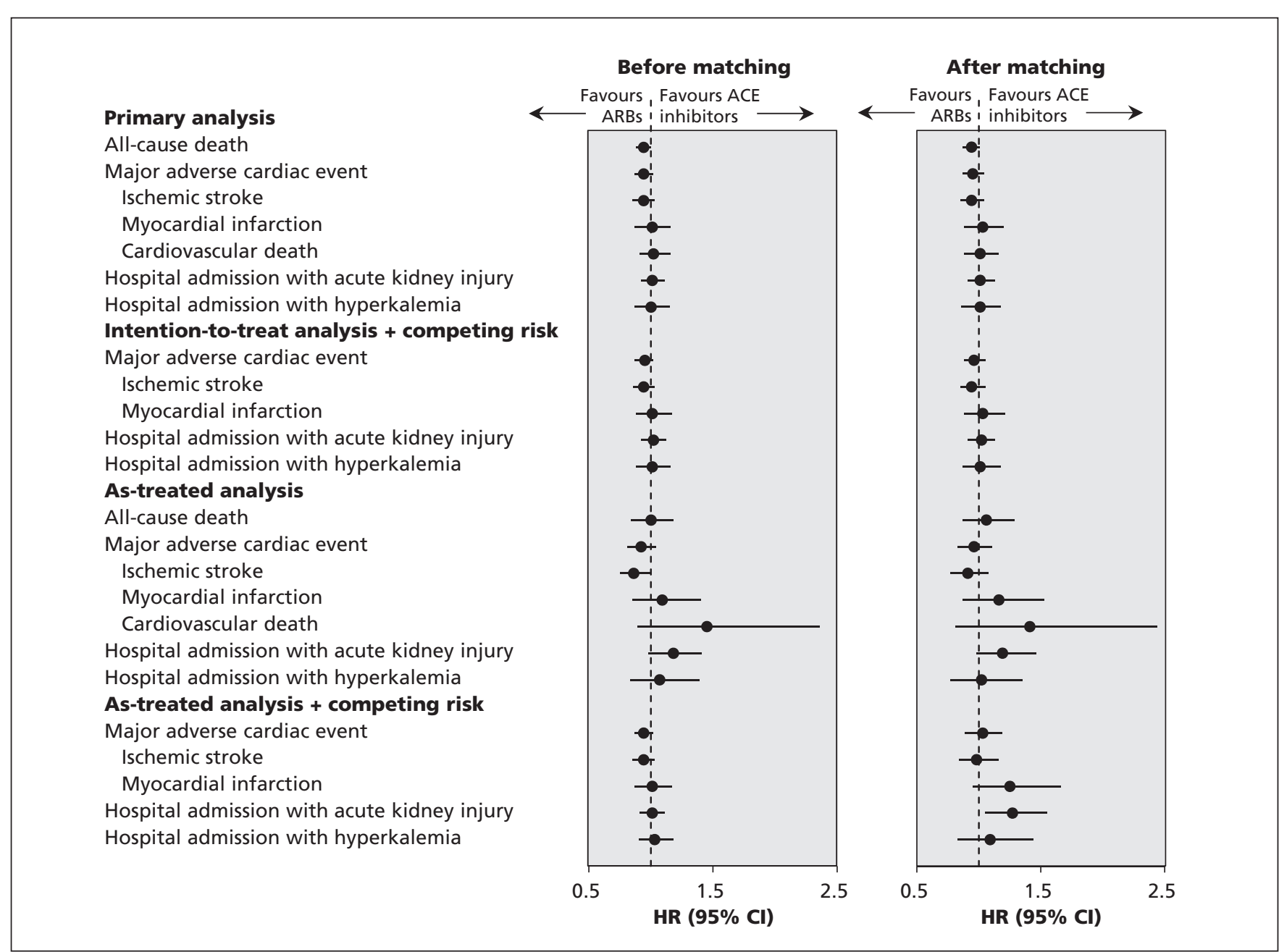

Figure 3: Sensitivity analyses for risk of primary and secondary outcomes among patients using angiotensin-converting-enzyme (ACE) inhibitors and angiotensin II receptor blockers (ARBs). $\mathrm{Cl}$ = confidence interval, $\mathrm{HR}$ = hazard ratio. 
reported. In a systematic review of 63 trials involving patients with diabetes and varying albuminuria, $\mathrm{Wu}$ and colleagues ${ }^{14}$ produced findings similar to those of our study. By contrast, another systematic review of 35 RCTs $^{15}$ showed that ACE inhibitors reduced all-cause mortality, cardiovascular-related mortality and major cardiovascular events in diabetes populations compared with ARBs, but the ONTARGET trial was not included in the analysis.

Given the inherent indirectness of comparison in meta-analyses and the lack of analyses focused specifically on patients with diabetic retinopathy, the findings from our large study add to the growing evidence of no significant difference between ACE inhibitors and ARBs in terms of incident macrovascular events in patients with diabetic retinopathy.

\section{Strengths and limitations}

The strengths of our study include the examination of a nationwide, population-based cohort using registry data from Taiwan's National Health Insurance program, which contains data on ophthalmologist-confirmed diabetic retinopathy, the prospective recording of subsequent major adverse cardiac events during a long follow-up period and the detailed examination of potential confounders. However, the study has several limitations. First, the diagnosis of diabetic retinopathy was based on the presence of the ICD-9-CM code in records; its severity and regression or progression, according to the Early Treatment Diabetic Retinopathy Study (ETDRS) scale, were not recorded in the database. Nevertheless, in a substudy of the Action to Control Cardiovascular Risk in Diabetes (ACCORD) trial, ${ }^{32}$ the risks of future macrovascular events did not appear to be graded according to the severity of diabetes.

Second, patients were not randomly assigned to the study groups. Thus, RCTs are needed to confirm or refute our findings.

Third, unmeasured bias is an inherent limitation of retrospective observational studies, although we conducted propensity score matching to address imbalances between groups.

Fourth, some patient data, including hemoglobin $\mathrm{A}_{1 \mathrm{C}}$ concentration, blood pressure and reasons underlying the selection of specific antihypertensive medications, were not available in the database. However, we balanced the types of antidiabetic drugs and non-renin-angiotensinaldosterone system antihypertensive medications in both groups.

Fifth, patients taking ACE inhibitors have more adverse symptoms (e.g., cough) than patients taking ARBs, mainly because of the unop- posed elevation of bradykinin, which may lead to intolerance to ACE inhibitors or even early discontinuation. Thus, the real effects of ACE inhibitors may be overestimated in intention-to-treat analyses, which may otherwise better represent real-life medical practice. Nevertheless, the results were consistent with those of the as-treated analyses, which we performed to reduce dropout bias.

Additionally, because ACE inhibitors and ARBs are preferred for the treatment of hypertension in patients with type 2 diabetes, prevalent use of these drugs before the diagnosis of diabetic retinopathy was not uncommon and may have led to prevalent user bias in our study. ${ }^{33}$ However, both patient groups had similar rates of prevalent use of the drugs. When we excluded prevalent users to minimize this bias, we found results consistent with those of the primary analyses. Thus, we believe that prevalent user bias is less likely to influence the validity of our study.

Finally, generalizability of our results to the diabetic patients without diabetic retinopathy may be difficult because of the varied time lapse between the diagnosis of type 2 diabetes and diabetic retinopathy in retrospective claims analyses.

\section{Conclusion}

In this nationwide population-based cohort of patients with diabetic retinopathy, we found that use of ARBs and ACE inhibitors was comparable in terms of risk of all-cause death, major adverse cardiac events and adverse effects. Our findings support the existing evidence that ACE inhibitors and ARBs are equally effective and safe in this patient population, which may aid treatment decisions based on individuals' adherence or tolerance.

\section{References}

1. Rosenson RS, Fioretto P, Dodson PM. Does microvascular disease predict macrovascular events in type 2 diabetes? Atherosclerosis 2011;218:13-8.

2. Cheung N, Wang JJ, Klein R, et al. Diabetic retinopathy and the risk of coronary heart disease: the Atherosclerosis Risk in Communities Study. Diabetes Care 2007;30:1742-6.

3. Kramer CK, Rodrigues TC, Canani LH, et al. Diabetic retinopathy predicts all-cause mortality and cardiovascular events in both type 1 and 2 diabetes: meta-analysis of observational studies. Diabetes Care 2011;34:1238-44.

4. Sjølie AK, Klein R, Porta M, et al. Effect of candesartan on progression and regression of retinopathy in type 2 diabetes (DIRECT-Protect 2): a randomised placebo-controlled trial. Lancet 2008;372:1385-93.

5. Mohamed Q, Gillies MC, Wong TY. Management of diabetic retinopathy: a systematic review. JAMA 2007;298:902-16.

6. Mancia G, Fagard R, Narkiewicz K, et al. 2013 ESH/ESC guidelines for the management of arterial hypertension: the Task Force for the Management of Arterial Hypertension of the European Society of Hypertension (ESH) and of the European Society of Cardiology (ESC). Eur Heart J 2013;34:2159-219.

7. American Diabetes Association. Standards of medical care in diabetes - 2013. Diabetes Care 2013;36(Suppl 1):S11-66.

8. Heart Outcomes Prevention Evaluation Study Investigators. Effects of ramipril on cardiovascular and microvascular outcomes in people with diabetes mellitus: results of the HOPE study and MICRO-HOPE substudy. Lancet 2000;355:253-9. 
9. Tillin T, Orchard T, Malm A, et al. The role of antihypertensive therapy in reducing vascular complications of type 2 diabetes. Findings from the DIabetic REtinopathy Candesartan TrialsProtect 2 study. J Hypertens 2011;29:1457-62.

10. Brenner BM, Cooper ME, de Zeeuw D, et al. Effects of losartan on renal and cardiovascular outcomes in patients with type 2 diabetes and nephropathy. N Engl J Med 2001;345:861-9.

11. Lewis EJ, Hunsicker LG, Clarke WR, et al. Renoprotective effect of the angiotensin-receptor antagonist irbesartan in patients with nephropathy due to type 2 diabetes. $N$ Engl $J$ Med 2001;345:851-60.

12. Haller H, Ito S, Izzo JL Jr, et al. Olmesartan for the delay or prevention of microalbuminuria in type 2 diabetes. $N$ Engl $\mathrm{J} \mathrm{Med}$ 2011;364:907-17.

13. Yusuf S, Diener HC, Sacco RL, et al. Telmisartan to prevent recurrent stroke and cardiovascular events. N Engl J Med 2008; 359:1225-37.

14. Wu HY, Huang JW, Lin HJ, et al. Comparative effectiveness of renin-angiotensin system blockers and other antihypertensive drugs in patients with diabetes: systematic review and bayesian network meta-analysis. BMJ 2013;347:f6008.

15. Cheng J, Zhang W, Zhang X, et al. Effect of angiotensinconverting enzyme inhibitors and angiotensin II receptor blockers on all-cause mortality, cardiovascular deaths, and cardiovascular events in patients with diabetes mellitus: a meta-analysis. JAMA Intern Med 2014;174:773-85.

16. ONTARGET Investigators, Yusuf S, Teo KK, Dyal, L, et al Telmisartan, ramipril, or both in patients at high risk for vascular events. N Engl J Med 2008;358:1547-59.

17. Shih CJ, Chu H, Chao PW, et al. Long-term clinical outcome of major adverse cardiac events in survivors of infective endocarditis: a nationwide population-based study. Circulation 2014;130: 1684-91.

18. Chao PW, Shih CJ, Lee YJ, et al. Association of postdischarge rehabilitation with mortality in intensive care unit survivors of sepsis. Am J Respir Crit Care Med 2014;190:1003-11.

19. Lin CC, Lai MS, Syu CY, et al. Accuracy of diabetes diagnosis in health insurance claims data in Taiwan. J Formos Med Assoc 2005; $104: 157-63$

20. Early Treatment Diabetic Retinopathy Study Research Group. Grading diabetic retinopathy from stereoscopic color fundus photographs - an extension of the modified Airlie House classification [ETDRS report no. 10]. Ophthalmology 1991;98:786-806.

21. Early Treatment Diabetic Retinopathy Study Research Group. Fundus photographic risk factors for progression of diabetic retinopathy [ETDRS report no. 12]. Ophthalmology 1991;98: 823-33.

22. Chang HY, Weiner JP, Richards TM, et al. Validating the adapted Diabetes Complications Severity Index in claims data. Am J Manag Care 2012;18:721-6.

23. Cheng CL, Kao YH, Lin SJ, et al. Validation of the National Health Insurance Research Database with ischemic stroke cases in Taiwan. Pharmacoepidemiol Drug Saf 2011;20:236-42.

24. Cheng CL, Lee CH, Chen PS, et al. Validation of acute myocardial infarction cases in the National Health Insurance Research database in Taiwan. J Epidemiol 2014;24:500-7.

25. Fine JP, Gray RJ. A proportional hazards model for the subdistribution of a competing risk. J Am Stat Assoc 1999;94:496-509.

26. Wang B, Wang F, Zhang Y, et al. Effects of RAS inhibitors on diabetic retinopathy: a systematic review and meta-analysis. Lancet Diabetes Endocrinol 2015;3:263-74.

27. Muirhead N, Feagan BF, Mahon J, et al. The effects of valsartan and captopril on reducing microalbuminuria in patients with type 2 diabetes mellitus: a placebo-controlled trial. Curr Ther Res 1999;60:650-60.
28. Tütüncü NB, Gürlek A, Gedik O. Efficacy of ACE inhibitors and ATII receptor blockers in patients with microalbuminuria: a prospective study. Acta Diabetol 2001;38:157-61.

29. Ko GT, Tsang CC, Chan HC. Stabilization and regression of albuminuria in Chinese patients with type 2 diabetes: a one-year randomized study of valsartan versus enalapril. Adv Ther 2005; 22:155-62.

30. Barnett AH, Bain SC, Bouter P, et al. Angiotensin-receptor blockade versus converting-enzyme inhibition in type 2 diabetes and nephropathy. N Engl J Med 2004;351:1952-61.

31. Lacourcière Y, Bélanger A, Godin C, et al. Long-term comparison of losartan and enalapril on kidney function in hypertensive type 2 diabetics with early nephropathy. Kidney Int 2000;58:762-9.

32. Mottl AK, Pajewski N, Fonseca V, et al. The degree of retinopathy is equally predictive for renal and macrovascular outcomes in the ACCORD Trial. J Diabetes Complications 2014;28:874-9.

33. Ray WA. Evaluating medication effects outside of clinical trials: new-user designs. Am J Epidemiol 2003;158:915-20.

Affiliations: School of Medicine (Shih, H.-T. Chen, Kuo, Li, Y.-T. Chen), National Yang-Ming University, Taipei; Department of Medicine (Shih), Taipei Veterans General Hospital, Yuanshan Branch, Yuanshan, Taiwan; Division of Endocrinology and Metabolism (H.-T. Chen), Department of Medicine, Taipei City Hospital, Heping Fuyou Branch, Taipei; National Institute of Infectious Diseases and Vaccinology (Kuo), National Health Research Institutes, Zhunan, Taiwan; Divisions of Infectious Diseases (Kuo) and Nephrology (Li, Ou), Department of Medicine, Taipei Veterans General Hospital, Taipei; Department of Nursing (Lai), Taipei Veterans General Hospital, Yuanshan Branch, Yuanshan; Department of Nursing (S.-C. Chen), and Division of Nephrology (Y.-T. Chen), Department of Medicine, Taipei City Hospital, Heping Fuyou Branch, Taipei, Taiwan

Contributors: Chia-Jen Shih and Hung-Ta Chen contributed equally as joint first authors. Chia-Jen Shih, Hung-Ta Chen, Shuo-Ming Ou and Yung-Tai Chen contributed to the study concept and design. Chia-Jen Shih, Hung-Ta Chen, Shuo-Ming $\mathrm{Ou}$ and Yung-Tai Chen contributed to the collection and assembly of data. Chia-Jen Shih, Szu-Yuan Li, Shuo-Ming Ou, Yung-Tai Chen provided statistical expertise, and Chia-Jen Shih, Hung-Ta Chen, Shu-Chen Kuo, Szu-Yuan Li, Pi-Hsiang Lai, Shu-Chen Chen and Yung-Tai Chen contributed to the analysis and interpretation of the data. Chia-Jen Shih, Hung-Ta Chen, Shuo-Ming Ou and Yung-Tai Chen drafted the manuscript. All of the authors revised the manuscript critically for important intellectual content, approved the final version to be published and agreed to act as guarantors of the work.

Funding: This study was supported in part by grants from Taipei Veterans General Hospital (no. V105A-003).

Acknowledgement: This study was based in part on data from the National Health Insurance Research Database provided by the Bureau of National Health Insurance (BNHI) of the Department of Health and managed by the National Health Research Institute. The conclusions presented in this article are those of the authors and do not necessarily reflect the views of the BNHI, the Department of Health or the National Health Research Institute. 